\title{
Controller Synthesis By Petri Nets Modeling
}

\author{
Abbas Dideban* \& Hassane Alla.** \\ * Semnan University, IRAN (Tel: (+98)231-3354123 \\ ** GIPSA lab, 38402 St Martin d'Heres Cedex FRANCE, \\ e-mail: adideban@Semnan.ac.ir, Hassane.alla@inpg.fr
}

\begin{abstract}
In this paper, we present two different methods for the synthesis of a simplified controller using Petri Nets. The supervisory control theory presented by Ramadge and Wonham is adapted to Petri nets modeling. Uncontrollable transitions in discrete event systems are the cause of forbidden states in supervisory control. This paper concerns the problem of forbidden states in safe Petri Net. We present different methods to reduce the number of constraints that prevent from forbidden states. Using these methods, we can construct a maximally permissive controller. The implementation of these approaches is considered using the SFC model.
\end{abstract}

Keywords: Controller, Discrete event system, Forbidden states, Linear constraints, Petri Nets.

\section{INTRODUCTION}

Discrete-event systems (DES) are becoming more and more complex and highly automated making it tricky the realization of an efficient and realistic control system. Given a discrete-event model of the plant and the specification of the desired behavior, the objective is to synthesize appropriate supervisor that will act in closed-loop with the plant according to the desired behavior. Ramadge and Wonham $(1987,1989)$ proved the existence and the characterization of the maximally permissive control. Finite-state machines and formal languages are the modeling framework considered in this study and the control action is achieved by event feedback. The main limitation in such an approach is the lack of structure in controlled automata.

Petri nets (PN) have been proposed as an alternative modeling formalism for DES control. Li and Wonham (1994) have presented an algorithm, which calculates the optimal solution for nets whose uncontrollable subnets are loop-free. The theory of regions in (Ghafari et al.,2003) allows the design of a maximally permissive PN controller. However, the number of control places is equal to the number of forbidden states and sometimes this leads to complex solutions. Holloway and Krogh (1990) have presented a method for calculating in real time a controller for a safe and cyclic marked graph. In (Holloway et al.,1996) the authors give a solution in a general case while complex controls are associated with transitions.

In this paper, we have a brief presentation of several new methods for controller synthesis by safe Petri Net. In these approaches, we used the principal idea of supervisory control that was presented by Ramadge and Wonham adapted to Petri nets. The synchronized composition between process and specification models, gives the closed loop desired model. However, due to uncontrollable transition, it is possible that some transitions violate the rules of Petri Nets for firing a transition. Then there are states enabled by the process but by firing the uncontrollable transition, a non-authorized state is reached. These states also are called forbidden states. We use two methods for preventing from forbidden states. In the first approach, we use the linear constraints. By the method presented in (Giua et al. 1992), it is possible to use the linear constraints for preventing the forbidden states. The problem is that generally, the number of forbidden states is large and we arrive to a complex controller. In this paper, we present the methods for simplifying the controller. By using the method presented by Yamalidou (Yamalidou et al. 1996), we can calculate the control places. 
In the second method, we use the predicate for preventing from forbidden states that is similar to the method used in (Holloway and Krogh,1990). Moreover, we simplify these predicates to obtain a very simplified controller.

The main disadvantage of these approaches is the calculation of the marking graph as in intermediate step, fortunately done off line.

This paper is organized as follows: In the second section, the fundamental definitions will be presented. In Section 3, the idea of passage from forbidden states to the linear constraints will be introduced. In section 4, the principal idea of controller synthesis and different methods for this will be presented. The simplification method will be presented in section 5. In Section 6, we explain how we could transfer the final PN model into a SFC model which is directly implementable on a PLC (Programmable Logic Controller). Finally, the conclusion is given in the last section.

\section{FUNDEMENTAL DEFINITIONS}

In this paper, it is supposed that the reader is familiar with the bases of the Petri net paradigm (David and Alla 2005) and the concept of discrete events systems supervisory (Wonham 2005). We present only the notions used here.

A PN is presented by a 5-uple $N=\{P, T, W, C, B\}$ where: 1) $P$ is the set of places, 2) $T$ is the set of transitions, 3) W: $(P \times T) \cup(P \times T)$, is the incidence matrix, 4) $C$ is the conditions of firing for each controllable transition, and 5) $B:(C \times T)$ is the set of directed arcs associating control places with transitions(Holloway and Krogh,1990).

Safe PNs are considered here. The reachability graph consists of nodes, which correspond to the accessible markings $M_{i}$, and arcs to the firing of the transitions. In the reachability graph, there are two types of states: the authorized state $M_{A}$ and the forbidden state $M_{F}$. Among the forbidden states, a particular and important subset is constituted by the border forbidden states, which are denoted by the set $M_{B}$. These states are such that all the input transitions are controllable.

In this paper, we use the word state instead of marking.

Definition 1: The set $\{0,1\}^{N}$ represents all the Boolean vectors of dimension $N$.

A marking of PN model by $N$ places is a vector of set $\{0,1\}^{N}$.

The set of the marked places of a marking $M$ is given by a Support function that will be defined in the following.

Definition 2: The function Support $(X)$ of a vector $X \in\{0,1\}^{N}$ is presented as below:

Support $(X)=$ The set of marked places in vector $X$.

The support of vector $M_{0}^{\top}=[1,0,1,0,0,1,0]$ is presented as bellow:

$$
\text { Support }\left(M_{0}\right)=\left\{P_{1} P_{3} P_{6}\right\}
$$

Definition 3: Let $M_{1}=\left(P_{11} P_{12} P_{13} \ldots P_{1 n}\right)$ be a state of the system. $M_{2}=\left(P_{21} P_{22} P_{23} \ldots P_{2 m}\right)$ is an overstate of $M_{1}$ if:

$\forall i \in[1, n], \exists j \in[1, m]$ such that $P_{1 i}=P_{2 j}$

This relation is represented as shown below:

$$
M_{2} \subset M_{1}
$$

The forbidden states that are used in this paper are divided in to two groups. The first group corresponds to the forbidden states of the classical approach developed by Ramadge and Wonham. In this approach, the model of the process is synchronized with the specification which gives the closed loop desired functioning. The existence of uncontrollable transitions often leads to the existence of the forbidden states including the border forbidden states. Deadlock states constitute the second group of forbidden states. Both types of the forbidden states are considered in the same way.

The border forbidden states are reached from the admissible states by the occurrence of controllable events. Preventing the occurrence of the controllable events can forbid entering to a forbidden state. By constructing the reachability graph, we can divide the authorized states for each 
controllable event into two groups. We present these states as bellow:

Definition 4: Let $M_{B}$ be the set of border forbidden states and $M_{A}$ the set of admissible states. The sets of $t_{i}$ critical states $M_{t_{i}}^{C}$, and $t_{i}$ sound states $M_{t_{i}}^{S}$, are defined as follows:

$$
\begin{aligned}
\forall t_{i} \in \sum_{\mathrm{c}} M_{t_{i}}^{C}=\left\{m_{i} \in M_{A} \mid m_{i} \stackrel{t_{i}}{\longrightarrow} m_{j}, m_{j} \in M_{B}\right\} \\
M_{t_{i}}^{S}=\left\{m_{i} \in M_{A} \mid m_{i} \longrightarrow t_{i} \longrightarrow m_{j}, m_{j} \in M_{A}\right\}
\end{aligned}
$$

Where $\Sigma_{\mathrm{c}}$ is the set of controllable transitions

The $t_{i}$ critical states are the states from which by firing transition $t_{i}$, the border forbidden states can be reached. The $t_{i}$ sound states are the states from which by firing transition $t_{i}$, the admissible states can be reached. Some time we can have for transition $t_{i}, M_{t_{i}}^{C}=\Phi$. In this case, it is not necessary to use the control for this transition.

Our approach is applicable on safe Petri Net, which is the common control model.

In this paper, it is supposed that all of the events are independent.

\section{FROM FORBIDDEN STATES TO LINEAR CONSTRAINTS}

Let $M_{i}\left(M_{i}^{\top}=\left[m_{i 1}, m_{i 2}, \ldots, m_{\mathbb{N}}\right]\right)$ a forbidden state ${ }^{1}$, in set $M_{B}$ and Support $\left(M_{i}\right)=\left\{P_{i 1} P_{i 2} P_{i 3} \ldots P_{i n}\right\}$ the set of marked places of $M_{i}$. From a forbidden state, a linear constraint can be constructed ( Giua et al. 1992).

The linear constraint deduced from the forbidden state $M_{i}$ is given below. The state $M_{i}$ does not verify this relation thus by applying this relation, it will be forbidden.

$$
\sum_{k=1}^{n} m_{i k} \leq n-1
$$

Where $n=$ Card [Support $\left(M_{i}\right)$ is the number of marked places of $M_{i}$, and $m_{i k}$ the marking of place $P_{i k}$ of state $M_{i}$.

Let $M\left(M^{\top}=\left[m_{1}, m_{2}, \ldots, m_{N}\right]\right)$ be a general marking and $M_{i}$ be a forbidden state. The constraint forbidding state $M_{i}$ is denoted as $c_{i}$ and can be rewritten in the following form:

$$
\left.M_{i}^{\top} . M \leq \text { Card [Support }\left(M_{i}\right)\right]-1
$$

For example if:

$M_{i}^{\top}=[0,1,1,0,0,0,1] \Rightarrow$ Card [Support $\left.\left(M_{i}\right)\right]=3 \quad \Rightarrow \quad m_{2}+m_{3}+m_{7} \leq 2$

Verifying relation 1 is equivalent to forbid state $M_{i}$ when the PN model is conservative. However, in a safe PN not necessary conservative, this equivalence is not always true. This problem will be discussed later.

This equivalence is necessary to obtain the optimal supervisor, but it is always possible to simplify the constraints. In our approach, this property will be examined in the properties of simplification.

In this paper the forbidden state and its constraint is presented as below:

$$
\begin{aligned}
& f_{\mathrm{i}}=\left\{P_{\mathrm{i} 1} P_{\mathrm{i} 2} P_{\mathrm{i} 3} \ldots P_{\mathrm{in}}\right\} \\
& C_{i}=\left\{P_{\mathrm{i} 1} P_{\mathrm{i} 2} P_{\mathrm{i} 3} \ldots P_{i n}, n-1\right\}
\end{aligned}
$$

The last term in $c_{i}$ corresponds to the number of marked places in the forbidden state minus 1 , it is called the bound. This is true only at the beginning.

\section{CONTROLLER SYNTHESIS BY PN MODELLING}

There are several ways to specify the constraints for the control synthesis.

- Using PN model. (when the specification is a sequential process)

\footnotetext{
${ }^{1}$ Where there is no ambiguity, the word border will be omitted.
} 
- Giving directly the constraints upon the markings

- Using a PN model with inhibitors and incident arcs

- Giving directly the set of forbidden states

If all of the transitions are controllable, the controller synthesis is easy. The synchronized composition between the specification model and the process model gives the implementable controller. However, generally, there are uncontrollable transitions and the closed loop behavior is controllable. A synthesis procedure must applied.

To illustrate the ideas developed in this paper, let us consider the example presented in (Dideban and Alla 2005). A manufacturing system is composed of two independent machines $M_{1}$ and $M_{2}$, two transfer robots of parts and one test bench where the final products are tested (Figure 2).

Each machine has the following operating cycle: By occurrence of the controllable event $c_{i}$, the machine starts working. When the work is finished (occurrence of uncontrollable event $f w m_{i}$ ) the robot transfers the produced part on the assembly station, and a new cycle can be started again by occurrence of the uncontrollable event $\mathrm{ftm}_{i}$.

$\Sigma_{c}=\left\{c_{1}, c_{2}\right\}$ et, $\Sigma_{u}=\left\{f w m_{1}, f t m_{1}, f w m_{2}, f t m_{2}\right\}$.

The PN model of the process and specification are given in Figure 3 . The specification enforces that the robot transfers first the product of machine $M_{1}$ and then the product of machine $M_{2}$.

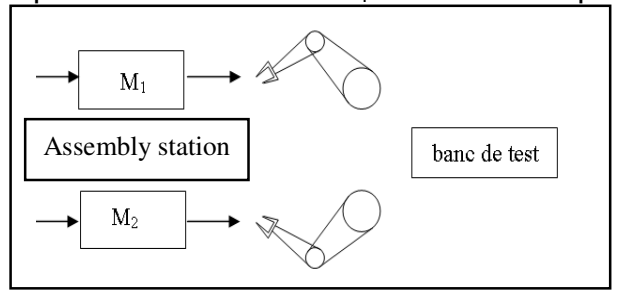

Fig. 2: Production line
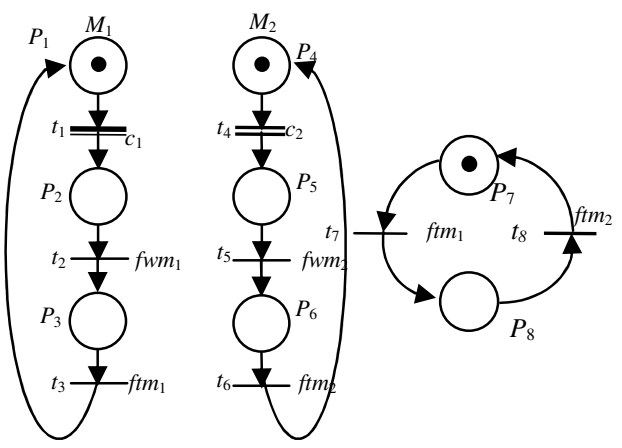

$M_{1} \quad M_{2} \quad$ Specification

Fig. 3: Process and specification model.

\subsection{Forbidden states}

The determination of the forbidden states is made by the study of the controllability of the language of the specification with regard to the language generated by the model of the desired functioning in closed loop. The closed loop model of this system is illustrated in Figure 4 and will be denoted as $R_{\mathrm{d}}$. In this model transition $t_{3}$ and $t_{6}$ cannot always respect the PN firing rules. For example, when place $P_{3}$ is marked and place $P_{7}$ is not marked, the firing of transition $t_{3}$ cannot be forbidden since this transition is not controllable. It is not possible to stop the event. ftm $_{1}$ These states are called forbidden states. By Kumar algorithm (Kumar and Holloway 1996) we can calculate this set and 
from this the set of border forbidden states called $M_{B}$.

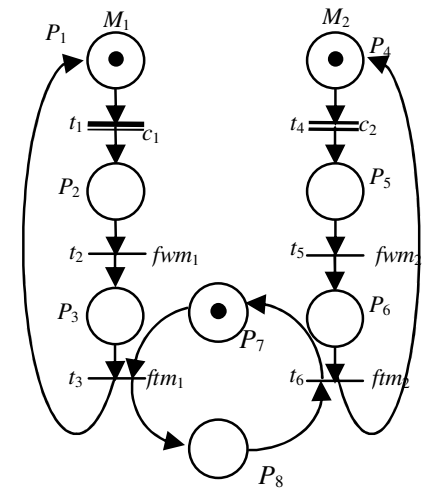

Fig. 4: Petri net model of closed loop system

$$
M_{B}=\left\{\left(P_{7} P_{5} P_{1}\right),\left(P_{7} P_{5} P_{2}\right),\left(P_{7} P_{5} P_{3}\right),\left(P_{8} P_{4} P_{2}\right),\left(P_{8} P_{5} P_{2}\right),\left(P_{8} P_{6} P_{2}\right)\right\}
$$

From the set of border forbidden states, we can determine the set of constraints that can prevent reaching this set (Giua 1992).

$m_{7}+m_{5}+m_{1} \leq 2 \quad m_{7}+m_{5}+m_{2} \leq 2 \quad m_{7}+m_{5}+m_{3} \leq 2$

$m_{8}+m_{4}+m_{2} \leq 2 \quad m_{8}+m_{5}+m_{2} \leq 2 \quad m_{8}+m_{6}+m_{2} \leq 2$

\subsection{Calculation of control places}

To calculate control places for each linear constraint, there is a systematic method given by (Yamalidou et al. 1996). The incidence matrix of PN $R_{\mathrm{d}}$, corresponding to the closed loop functioning is $W_{p}$. The synthesis approach consists in adding to the incidence matrix $W_{p}$ a matrix $W_{c}$ corresponding to the each control place. The incidence matrix of controlled system $W$ will be constructed by adding a line to the incidence matrix of the controlled process $W_{p}$ for each control place.

$$
W=\left[\begin{array}{l}
W_{p} \\
W_{c}
\end{array}\right]
$$

It is then possible to calculate the matrix $W_{c}$ and its initial marking as bellow, where $L$ represents the sets of constraints.

$$
\begin{aligned}
& W_{c}=-L . W_{P} \\
& M_{\text {cinit }}=b-L \cdot M_{\text {pinit }}
\end{aligned}
$$

The controller is known by the determination of its incidence matrix $W_{c}$ and its initial marking $M_{\text {cinit. }}$.

The initial marking of the control places is calculated:

$M_{c 0}{ }^{\top}=[0,1,1,1,2,2]$

$$
L=\left[\begin{array}{llllllll}
1 & 0 & 0 & 0 & 1 & 0 & 1 & 0 \\
0 & 1 & 0 & 0 & 1 & 0 & 1 & 0 \\
0 & 0 & 1 & 0 & 1 & 0 & 1 & 0 \\
0 & 1 & 0 & 1 & 0 & 0 & 0 & 1 \\
0 & 1 & 0 & 0 & 1 & 0 & 0 & 1 \\
0 & 1 & 0 & 0 & 0 & 1 & 0 & 1
\end{array}\right]
$$

By applying this result to the example, the result is: 


$$
W c=\left[\begin{array}{cccccc}
1 & 0 & 0 & -1 & 1 & -1 \\
-1 & 1 & 1 & -1 & 1 & -1 \\
0 & -1 & 2 & -1 & 1 & -1 \\
-1 & 1 & -1 & 1 & 0 & 0 \\
-1 & 1 & -1 & -1 & 1 & 1 \\
-1 & 1 & -1 & 0 & -1 & 2
\end{array}\right]
$$

This technique gives the controller in an elegant way. However, there are some disadvantages:

-The final model is very complicated since the number of control places can be very large.

- The safe PN model is changed to a non safe model.

We will present below simplification methods which will solve these problems and give a simple controller.

\subsection{Calculation of forbidding conditions}

The first method that we propose is to add conditions to the controllable transitions (Dideban and Alla 2006).

From the set of critical states and for each controllable transition, we calculate the conditions associated with this transition. By adding the controls as described below, we can forbid the firing of this transition towards the forbidden states.

Definition 5: Let $M_{t_{i}}^{C}$ be the set of critical states for transition $t_{i}$. Control $U t_{i:}\left(M_{t_{i}}^{C}, M_{j}\right) \rightarrow\{0,1\}$ is defined as follow:

$$
U_{t_{i}}\left(\mathrm{M}_{\mathrm{t}_{\mathrm{i}}}^{\mathrm{C}}, M_{j}\right)=\left\{\begin{array}{lr}
0 & \mathrm{M}_{\mathrm{j}} \in \mathrm{M}_{\mathrm{t}_{\mathrm{i}}}^{\mathrm{C}} \\
1 & \text { if not }
\end{array}\right.
$$

Adding this control is indicated in figure 5.

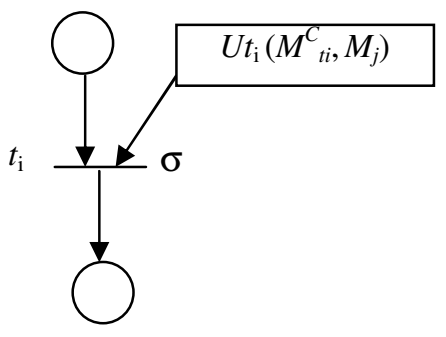

Fig. 5. Adding the condition as a controller

Nevertheless, how we can determine analytically the logic expression $U_{t i}\left(M_{j}\right)$ in relation with state $M_{j}$. This is done simply in the following way.

$M_{j}=P_{1} P_{4} P_{7} \Rightarrow U_{t i}\left(M_{j}\right)=\left(m\left(P_{1}\right) \& m\left(P_{4}\right) \& m\left(P_{7}\right)\right)^{\prime}$

If the number of critical states is more than one, the $O R$ operation between them gives the control. This idea is presented via the next properties.

Corollary 1: Let $M_{1}$ be a critical state for transition $t_{i}$. By using the control $U t_{i}\left(M_{1}\right)$, we can forbid the firing of $t_{i}$ in state $M_{1}$.

$$
U_{t_{i}}\left(M_{1}\right)=\left(\prod_{r=1}^{\operatorname{card}\left(\text { sup port }\left(M_{1}\right)\right.}\left(P_{1 r}\right)\right)^{\prime}
$$

By this method, it is possible to forbid the firing from state $M_{1}$, For constructing a maximal permissive controller, the modification of the conditions will be considered in the next property.

Corollary 2: Let $M_{t_{i}}^{C}=M_{1}, M_{2}, \ldots, M_{m}$, be the set of all the $t_{i}$ critical- states, the condition for forbidding the firing towards the forbidden states will be calculated as follows: 


$$
U_{t_{i}}\left(M_{t_{i}}^{C}\right)=\left(\bigcup_{k=1}^{\operatorname{card}\left(M_{t_{i}}^{C}\right)}\left(\prod_{r=1}^{\operatorname{card}\left(\sup \operatorname{port}\left(M_{k}\right)\right.} m\left(P_{k r}\right)\right)\right)^{\prime}
$$

Now, the condition of firing can be calculated for each transition. However, sometimes the conditions are complex and makes very difficult to understand the dynamic behavior of the system. Moreover, the calculation time for the conditions in real time can be very large. It is then necessary to use similar simplification methods presented in (Dideban and Alla 2008).

\section{SIMPLIFICATION OF THE CONTROLLER}

To solve the complexity problem of the controller, we present three methods for simplification. In the first method by using the marking invariant, we reduce the number of constraints and also their bound (Dideban and Alla 2005).

In the second method, by presenting the new definition of over-state, we simplify the number of constraints (Dideban and Alla 2008).

In the third method by calculation of simplified predicate, it is possible to obtain a very simplified controller (Dideban and Alla 2006).

\subsection{First method: Simplification by marking invariant}

Let consider the previous example. We have 6 constraints that can be divided to two groups. Group 1:

$m_{7}+m_{5}+m_{1} \leq 2 \quad m_{7}+m_{5}+m_{2} \leq 2 \quad m_{7}+m_{5}+m_{3} \leq 2$

For the set of places $\left\{P_{1}, P_{2}, P_{3}\right\}$ we have the marking invariant: $m_{3}+m_{2}+m_{1}=1$

By adding the 3 constraints and using the marking invariant, we have:

$3\left(m_{7}+m_{5}\right)+\left(m_{1}+m_{2}+m_{3}\right) \leq 6 \quad \Rightarrow \quad m_{7}+m_{5} \leq 5 / 3$

The marking number is integer and we have: $m_{7}+m_{5} \leq 1$

For the other group also we obtain: $m_{8}+m_{2} \leq 1$

We can generalize this idea via 2 properties;

\section{Corollary 3 :}

Let $\left.\left(P_{1} P_{\mathrm{i} 1} \ldots P_{\mathrm{i}(\mathrm{n}-1)}\right), \ldots,\left(P_{\mathrm{r}} P_{\mathrm{i} 1} \ldots P_{\mathrm{i}(\mathrm{n}-1)}\right)\right\}$ be $r$ forbidden states in $M_{\mathrm{B}}$. And we have the marking invariant:

$$
m_{1}+m_{2}+\ldots+m_{\mathrm{r}}=1
$$

The $r$ constraints are equivalent to one constraint:

$$
\begin{aligned}
m_{1}+m_{i 1}+\ldots+m_{i(n-1)} & \leq n-1 \\
m_{2}+m_{i 1}+\ldots+m_{i(n-1)} & \leq n-1 \\
\ldots & \Longleftrightarrow \\
m_{\mathrm{r}}+m_{\mathrm{i} 1}+\ldots+m_{\mathrm{i}(n-1)} & \leq n-1
\end{aligned} \quad m_{\mathrm{i} 1}+\ldots+m_{\mathrm{i}(n-1)} \leq n-2
$$

Where $n$ is the number of marked place.

$$
\square
$$

Sometimes the number of constraints in a group is not exactly equal to the number of places in an invariant. For example suppose that we have: $m_{7}+m_{5}+m_{1} \leq 2 \quad m_{7}+m_{5}+m_{2} \leq 2$

Also we have $m_{3}+m_{2}+m_{1}=1$

From this invariant, we can construct a partial invariant such as: $m_{2}+m_{1} \leq 1$

It is possible simplify the two constraints by using partial invariant:

$m_{7}+m_{5}+m_{1} \leq 2 \quad m_{7}+m_{5}+m_{2} \leq 2 \Rightarrow m_{7}+m_{5}+m_{2}+m_{1} \leq 2$

\section{Corollary 4:}

Let $C=\left\{\left(P_{1} P_{i 1} \ldots P_{i(n-1)}, k\right),\left(P_{2} P_{i 1} \ldots P_{i(n-1)}, k\right), \ldots,\left(P_{r} P_{i 1} \ldots P_{i(n-1)}, k\right)\right\}$ be the equivalent constraints to forbidden states of marking graph and

$$
m_{1}+m_{2}+\ldots+m_{\mathrm{r}} \leq 1
$$

The $r$ constraints are equivalent to one constraint as below: 


$$
\begin{aligned}
m_{1}+m_{i 1}+\ldots+m_{i(n-1)} & \leq k \\
m_{2}+m_{i 1}+\ldots+m_{i(n-1)} & \leq k \\
\ldots & \Longleftrightarrow m_{1}+m_{2}+\ldots+m_{r}+m_{i 1}+\ldots+m_{i(n-1)} \leq k \\
m_{r}+m_{i 1}+\ldots+m_{i(n-1)} & \leq k
\end{aligned}
$$

Where $n$ is the number of marked place.

By using the second property, the number of constraint can be reduced but the constraint bound is not reduced. Then using property 1 is preferred. In a PN, there are often states that are not reachable from the initial making. However, these sates can verify marking invariants. These states can help us to reduce the bound of the constraint. This method is presented in [11].

We can use two properties to obtain the simplified constraint and finally we can select more simplified constraint via a method similar to the one presented by Mac Cluskey for logic expression( Moriss Mano M. 2001).

\subsection{Second method: Simplification by the over-state concept}

An over-state can represent a complete state or a part of this one. In the example of the two machines, $P_{2} P_{4} P_{7}$ is a complete state that represents the situation of both machines and the specifications. $P_{2} P_{4}$ is an over-state of this state that represents a partial state of the system. We have noted that a state can be forbidden by a linear constraint. In the same way, it is possible to forbid an over-state by its corresponding constraint.

The name "over-state" is used because the constraint corresponding to an over-state holds the state's constraint. For example, the constraint $m_{2}+m_{4} \leq 1$ that corresponds to the over-state $M_{1}=$ $P_{2} P_{4}$ holds both following constraints:

$$
\begin{aligned}
& m_{2}+m_{4}+m_{6} \leq 2 \\
& m_{2}+m_{4}+m_{7} \leq 2
\end{aligned}
$$

These two constraints forbid states $M_{2}=P_{2} P_{4} P_{6}$ and $M_{3}=P_{2} P_{4} P_{6} . P_{4} P_{6}$ is an over-state of both states $P_{2} P_{4} P_{6}$ and $P_{2} P_{4} P_{7}$ which could be verified by $M_{1} \leq M_{2}$ and $M_{1} \leq M_{3}$. Thus by using only the constraint $m_{2}+m_{4} \leq 1$, both states $M_{2}$ and $M_{3}$ will be forbidden. However, this reduction is not always simple. It is possible that the simplified constraint forbids also some authorized states. We present below a simplification method, which guarantees that the constraints forbid only the forbidden states.

Remark 1: There are two relations of inclusion, which operate in opposite directions: a set inclusion and a marking inclusion. Let $M_{1} \leq M_{2}$ :

1) The set of the marked places in the over-state $M_{1}$ is included in the set of the marked places in the state $M_{2}$.

2) The set of the markings covered by $M_{1}$ contains those covered by marking $M_{2}$.

Corollary 5: Let $M_{1}$ and $M_{2}$ be two vectors of $\{0,1\}^{\mathrm{N}}$, and $c_{1}$ and $c_{2}$ be two corresponding constraints. If $M_{1} \leq M_{2}\left(M_{1}\right.$ is an over-state of $\left.M_{2}\right)$ and $c_{1}$ is true, then $c_{2}$ is also true:

$$
\begin{aligned}
& M_{1} \leq M_{2} \text { and } c_{1}: M_{1}^{\top} . M \leq \operatorname{Card}\left[\operatorname{Support}\left(M_{1}\right)\right]-1 \\
& \Rightarrow c_{2}: \quad M_{2}^{\top} . M \leq \operatorname{Card}\left[\operatorname{Support}\left(M_{2}\right)\right]-1
\end{aligned}
$$

\subsubsection{Set of over-states}

We have noted that to forbid a state, it is enough to forbid its over-state, but which over-state? This question will be answered in the sequel. To achieve this goal, we need to construct the set of over-states for the forbidden states.

Firstly, we calculate the set of over-states for each state and then the union of all over- 
states gives the final set.

Definition 6: Let $M_{i}=\left\{P_{i 1} P_{i 2} P_{i 3} \ldots P_{i n}\right\}$ be a state of the system. The set of the over-states of $M_{i}$, denoted by $M_{i}^{\text {over }}$, is equal to the set of the subsets of $M_{i}$ without the empty set.

For example, the state $M_{1}=P_{1} P_{4} P_{6} P_{9}$ give:

$$
M_{1}{ }^{\text {over }}=\left\{P_{1}, P_{4}, P_{6}, P_{9}, P_{1} P_{4}, P_{1} P_{6}, P_{1} P_{9}, P_{4} P_{6}, P_{4} P_{9}, P_{1} P_{4} P_{6}, P_{1} P_{4} P_{9}, P_{1} P_{6} P_{9}, P_{4} P_{6} P_{9}, P_{1} P_{4} P_{6} P_{9}\right\}
$$

Among, the set of forbidden states in $M_{F}$, only the Border States have to be considered in the controller synthesis. Let $M_{B}$ be this set and $B_{1}$ be the set of over-states of $M_{B}$.

$$
\mathrm{B}_{1}=\bigcup_{i=1}^{\operatorname{Card}\left(M_{B}\right)} M_{i}^{\text {over }}
$$

\subsubsection{Building the reduced set of over-states}

It is possible to build two sets of over-states; a set of the authorized over-states $A_{1}$, and that of the forbidden states $B_{1}$. It is obvious that no over-state of $A_{1}$ must be forbidden. Thus it is necessary to remove from set $B_{1}$, all over-states which are in $A_{1}$. This gives set $B_{2}$ :

$$
B_{2}=B_{1} \backslash A_{1}
$$

Remark 2: From the implementation point of view, it is not necessary to construct $A_{1}$. The set $M_{A}$ is directly used.

Corollary 6: Let $B_{1}$ be the set of over-states of $M_{B}$ and $A_{1}$ be the set of over-states of $M_{A}$ and:

$$
B_{2}=B_{1} \backslash A_{1}
$$

The markings verifying the set of constraints $C_{2}$ (equivalent to $B_{2}$ ) correspond to the complete set of authorized states.

In set $B_{2}$, it often possible to find couple of states $M_{1}$ and $M_{2}$ such that $M_{1} \leq M_{2}\left(M_{1}\right.$ is an overstate of $M_{2}$ ). In that case, $M_{2}$ must be removed. It is a redundant state, and set $B_{3}$ is then defined formally as follows:

$$
B_{3}=B_{2}-\left\{M_{2 i} \in B_{2} \mid \exists M_{2 j} \in B_{2}, M_{2 i} \geq M_{2}\right\}
$$

$B_{3}$ is the minimal set of over-states to be forbidden.

For the example, from Figure 2, the sets of border forbidden states and authorized states are:

$M_{B=}\left\{\left(P_{7} P_{5} P_{1}\right),\left(P_{7} P_{5} P_{2}\right),\left(P_{7} P_{5} P_{3}\right),\left(P_{8} P_{4} P_{2}\right),\left(P_{8} P_{5} P_{2}\right),\left(P_{8} P_{6} P_{2}\right)\right\}$

$M_{A}=\left\{P_{1} P_{4} P_{7}, P_{2} P_{4} P_{7}, P_{3} P_{4} P_{7}, P_{1} P_{4} P_{8}, P_{1} P_{5} P_{8}, P_{1} P_{6} P_{8}\right\}$

Sets $A_{1}, B_{1}, B_{2}$ and $B_{3}$ are then calculated.

$B_{1}=M_{1}$ over $\cup M_{2}^{\text {over }} \cup M_{3}^{\text {over }} \cup M_{4}^{\text {over }} \cup M_{5}^{\text {over }} \cup M_{6}^{\text {over }}=\left\{P_{1}, P_{2}, P_{3}, P_{4}, P_{5}, P_{6}, P_{7}, P_{8}, P_{7} P_{5}\right.$, $P_{7} P_{1}, P_{5} P_{1}, P_{7} P_{2}, P_{5} P_{2}, P_{7} P_{3}, P_{5} P_{3}, P_{8} P_{4}, P_{8} P_{2}, P_{4} P_{2}, P_{8} P_{5}, P_{8} P_{6}, P_{6} P_{2},\left(P_{7} P_{5} P_{1}\right),\left(P_{7} P_{5} P_{2}\right)$, $\left.\left(P_{7} P_{5} P_{3}\right),\left(P_{8} P_{4} P_{2}\right),\left(P_{8} P_{5} P_{2}\right),\left(P_{8} P_{6} P_{2}\right)\right\}$

$B_{3}=\left\{\mathcal{R}_{1}, \mathrm{P}_{2}, \mathrm{R}_{3}, R_{4}, P_{5}, P_{8}, R_{7}, P_{8}, P_{7} P_{5}, P_{7} R_{1}, P_{5} \mathcal{R}_{1}, P_{7} R_{2}, P_{5} P_{2}, P_{7} \mathcal{R}_{3}, P_{5} P_{3}, P_{8} \mathcal{R}_{4}, P_{8} P_{2}, P_{4} R_{2}\right.$, $\left.P_{8} P_{5}, P_{8} P_{6}, P_{6} P_{2},\left(P_{7} P_{5} P_{1}\right),\left(P_{7} R_{5} P_{2}\right),\left(P_{7} R_{5} P_{3}\right),\left(P_{8} R_{4} P_{2}\right),\left(P_{8} P_{5} P_{2}\right),\left(P_{8} P_{6} P_{2}\right)\right\}=\left\{P_{7} P_{5}, P_{5} P_{2}, P_{5} P_{3}\right.$, $\left.P_{8} P_{2}, P_{6} P_{2}\right\}$

We do not need to consider all of the constraint in set $B_{3}$. The set $B_{4}$ is sufficient.

$B_{4}=\left\{P_{7} P_{5}, P_{8} P_{2}\right\}$

The result for our example is similar to the one calculated by the last method.

\subsection{Third method: Simplification of the conditions}


The simplification method presented in this section is different from the method presented in(Dideban and Alla 2008). It is based on the concepts of critical and sound states, which are reachable states, while the previous approach uses mainly the concepts of forbidden states.

Corollary 7:Let $M_{2}$ be an over-state of a $t_{i}$ critical state $M_{1}$. Forbidding the firing of transition $t_{i}$ by control $U t_{\mathrm{i}}\left(M_{2}\right)$ leads to forbid the firing of transition $t_{i}$ in state $M_{1}$.

$U t_{i}\left(M_{2}\right)=0$ and $M_{2}<M_{1} \Rightarrow$

Transition $t_{i}$ is non fireable from $M_{1}$

This property guarantees that transition $t_{i}$ is not fireable in state $M_{1}$ by using any over-state of $M_{1}$ in $t_{i}$ control. However, the condition deduced from an over-state may forbid the firing of transition $t_{i}$ in some of the $t_{i}$ sound states. This problem is formalized via the following property:

Corollary 8: Let $M_{t i}{ }^{C}$ be the set of $t_{i}$ critical states and $M_{t i}{ }^{S}$ the set of $t_{i}$ sound states such that $M_{1}$ $\in M_{t i}{ }^{C}$ and $M_{2}<M_{1}$.

The control $U t_{i}\left(M_{1}\right)$ can be replaced by the control $U t_{i}\left(M_{2}\right)$ if there is no state $M_{3} \in M_{t i}^{S}$ such that:

$$
M_{2}<M_{3}
$$

An over-state can often cover several critical states, and then the condition computed for this over-state gives the control for all these critical states. Then the forbidding condition will be simpler. Our objective is to find the over-states that cover the maximum number of critical states. To reach this goal, a set of all over-states for the $t_{i}$ critical- state is constructed and the $t_{i}$ sound states must be deleted from this set. This construction is similar to the one presented in (Dideban and Alla 2008).

Now we calculate the controller for our example by this method.

$$
\begin{aligned}
& M_{t 1}{ }^{C}=\left\{\left(P_{8} P_{4} P_{1}\right),\left(P_{8} P_{5} P_{1}\right),\left(P_{8} P_{6} P_{1}\right)\right\} \\
& M_{t 1} S=\left\{\left(P_{7} P_{4} P_{1}\right)\right\} \Rightarrow U t_{1}\left(M_{t 1} C^{C}=\left(m\left(P_{8}\right)\right)^{\prime}=\left(m\left(P_{7}\right)\right)\right. \\
& M_{t 2}{ }^{C}=\left\{\left(P_{7} P_{4} P_{1}\right),\left(P_{7} P_{4} P_{2}\right),\left(P_{7} P_{4} P_{3}\right)\right\} \\
& M_{t 2}{ }^{S}=\left\{\left(P_{8} P_{4} P_{1}\right)\right\} \Rightarrow U t_{2}\left(M_{t 2} C^{C}\right)=\left(m\left(P_{7}\right)\right)^{\prime}=\left(m\left(P_{8}\right)\right)
\end{aligned}
$$

\section{FROM PN MODEL TO SFC PROGRAM}

In order to build an implementable model on a PLC, we are going to use the SFC tool. It is a programming language for PLCs that is mostly used in industry for automation. It is derived from safe PNs. But some conditions must be fulfilled in order to transform a PN model directly to a SFC (or Grafcet) (Giua and DiCesare1993), Moreover, since SFC has a periodical functioning, it is possible that some independent events occur in the same cycle (apparent simultaneity). These conditions are verified in our example and the transformation is straightforward.

The SFC model for the controller obtained by the Yamalidou approach (6 constraints) is very complicated since the PN model is not safe and the number of constraints is very large. The controller obtained by the first and second method is indicated in figure 6 .

The difference between the two methods is relative to the arcs that exist in the second case. These arcs are indicated in figure 6 by dotted lines. For the third technique, it is sufficient to add the control $U t_{1}\left(M_{t 1}{ }^{C}\right)$ and $U t_{2}\left(M_{t 2}{ }^{C}\right)$ as the conditions for transitions $t_{1}$ and $t_{2}$.

For simplification, the situation of outputs is not mentioned in figure 6 . 


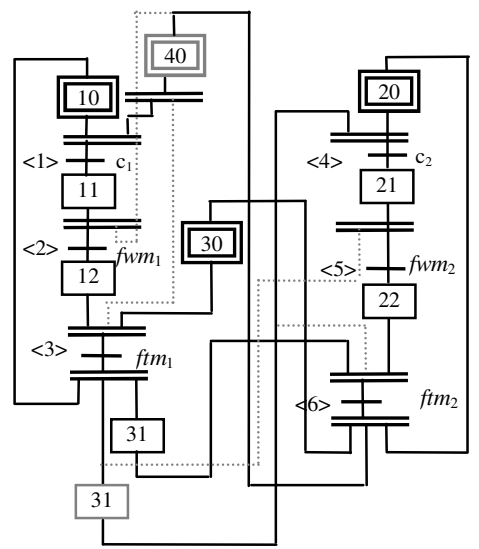

Fig. 6. Final controller by SFC

\section{CONCLUSION}

This paper presents an efficient approach for solving forbidden state control problems for a class of controlled discrete event systems, which can be modeled by safe Petri Nets. The basic idea is to use the simpler controller for preventing the forbidden states. This controller can be constructed by two approaches:

- by adding control places using the marking invariant or over-states)

- by adding conditions

The advantage of control places is that the dynamic of system is clear in the final model, but it is possible that the final model is not safe. When conditions are used, the model remains safe and it is possible to develop this method for non safe PN (Dideban and Alla 2009).

These methods are maximal permissive, since all of the used properties meet this condition. In controller synthesis by forbidding conditions, a dual method exists, if safe-states are considered instead of critical-states. Each one gives a maximal permissive controller and the simplest model must be chosen.

\section{REFERENCES}

David R., Alla H. (2005), Discrete, Continuous, and Hybrid Petri Nets, Springer.

Dideban, A., \& Alla, H. (2005). From forbidden state to linear constraints for the optimal supervisory control. Control Engineering and applied Informatics (CEAI), 7(3), 48-55.

Dideban A., Alla H., 2006, "Solving the problem of forbidden states by feedback control logical synthesis", The 32nd Annual Conference of the IEEE Industrial Electronics Society,7-10 Nov, Paris, FRANCE.

Dideban, A., \& Alla, H. (2008). Reduction of Constraints for Controller Synthesis based on Safe Petri Nets. Automatica, 44(7): 1697-1706.

Dideban A., Alla H., 2009, "Feedback control logic synthesis for non safe Petri nets", INCOM09, Jun 2009, Moscow, Russia.

Ghaffari A., Rezg N. and Xie X.-L. (2003). ", "Design of Live and Maximally Permissive Petri Net Controller Using Theory of Regions", IEEE Trans. On Robotics and Automation, vol 19, no 1, February.

Giua, A., DiCesare, F.M., \& Silva. (1992). Generalized Mutual Exclusion Constraints on Nets with Uncontrollable Transitions. In Proc. IEEE int. conf. on systems, man, and cybernetics (pp. 974-799).

Giua A., DiCesare F.(1993), "Grafcet and Petri Nets in Manufacturing", in Intelligent Manufacturing: Programming Environments for CIM, W.A. Gruver and J.C. Boudreaux (Eds.), pp. 153-76, Springer-Verlag. 
Holloway L. E., Guan X., Zhang L.(1996), "A Generalisation of state avoidance Policies for Controlled Petri Nets". IEEE Trans. Autom. Control, AC-41, 6, 804-816.

Holloway L. E., and Krogh B. H.( 1990), "Synthesis of feedback logic for a class of controlled Petri nets". IEEE Trans. Autom. Control, AC-35, 5, 514-523.

Kumar, R., \& Holloway, L.E. (1996). Supervisory control of deterministic Petri nets with regular specification languages. IEEE Trans. Automatic Control, 41(2):245-249.

Li Y., Wonham W. M. (1994), "Control of discrete-event systems-part ii: controller synthesis". IEEE Trans. Automatic Control, 39(3):512-531, Mars

Morris Mano, M. (2001). Digital design. Prentice Hall.

Ramadge, P.J., \& Wonham,W.M. (1987). Supervisory control of a class of discrete event processes. SIAM Joural of Control and Optimization, 25 (1):206-230.

Ramadge, P. J., \& Wonham, W. (1989). The control of discrete event systems.Dynamics of discrete event systems [Special issue]. Proceedings of thelEEE, 77(1), 81-98.

Wonham W. M (2005) , "Supervisory Control of Discrete-Event Systems", research report, http://www.control.toronto. edu/people/profs/wonham/wonham.html.

Yamalidou, K., Moody, J., Lemmon, M., \& Antsaklis, P. (1996). Feedback control of petri nets based on place invariants. Automatica, 32(1), 15-28. 